\title{
Leucémie et oncogènes : des facteurs de transcription hybrides
}

Les remaniements chromosomiques caractéristiques de types particuliers de tumeurs sont très fréquents chez l'homme, notamment dans les leucémies. Ces réarrangements peuvent activer des oncogènes ou, théoriquement du moins, inactiver des antioncogènes. L'activation d'oncogènes peut n'être que la conséquence d'une dérépression transcriptionnelle d'un proto-oncogène placé sous le contrôle de séquences stimulatrices fortes, comme cela est bien étudié pour le gène $c-m y c$ au cours des lymphomes de Burkitt [1]. Dans les leucémies myéloïdes chroniques, la translocation $\mathrm{t}(9 ; 22)$, caractéristique du chromosome Philadelphie, aboutit à la constitution d'un gène hybride, $b c r$ - $a b l$, qui est transcrit et traduit en un messager et une protéine également hybrides $\left(\mathrm{m} / \mathrm{s} n^{\circ} 7\right.$, vol. 1, p. 390). Il a très récemment été démontré que ce gène hybride, $b c r$-abl, était directement responsable de l'éclosion du syndrome myéloprolifératif $\left(\mathrm{m} / \mathrm{s} n^{\circ} 4\right.$, vol. 6 , p. 401). Le produit du gène abelson est une tyrosine kinase dont la fonction reste très mal connue mais qui semble activée après fusion avec la séquence $b c r$. Il est ainsi vraisemblable que la base du pouvoir oncogénique de la protéine $b c r-a b l$ est qu'elle transmet à la cellule un signal mitogène en l'absence de toute stimulation spécifique, comme cela est bien démontré dans le cas du produit de l'oncogène $c$-erb $B$, forme tronquée du récepteur du facteur de croissance $\operatorname{EGF}\left(\mathrm{m} / \mathrm{s} n^{\circ} 3\right.$, vol. 2, p. 151).

C'est maintenant la probable intervention dans une leucémie humaine d'un facteur de transcription hybride, engendré par remaniement chromosomique, que viennent de rapporter deux articles, l'un publié par des équipes américaines de Stanford, Irvine (CA) et Chicago (IL) [1], et l'autre du Whitehead Institute, Cam- bridge (MA) [2]. Il existe chez l'enfant une forme de leucémie aiguë lymphoblastique développée aux dépens de cellules pré-B dans laquelle une translocation $\mathrm{t}(1 ; 19)(\mathrm{q} 23 ; \mathrm{p} 13.3)$ est, presque constamment, retrouvée. Le chromosome 19 anormal possédant, au-delà de la bande 13.3 de son bras court, un fragment télomérique du bras long du chromosome 1 , situé au-delà de la bande 23 , est un marqueur de même valeur que le chromosome Philadelphie dans la LMC ou que la translocation t $(14 ; 18)$ dans le lymphome folliculaire $\left(\mathrm{m} / \mathrm{s} n^{\circ} 8\right.$, vol. 4, p. 515).

Le point de cassure en 19p13.3 passe par le gène $E 2 A$ codant pour des protéines se liant à l'ADN, appelées $\mathrm{E} 12$ et E47 (ces deux protéines ne diffèrent que de 60 acides aminés). E12/E47 se fixent au site E2 du enhancer du gène codant pour la chaîne légère d'immunoglobuline $\kappa$, ainsi qu'à des sites de même nature localisés dans le enhancer du gène codant pour les chaînes lourdes des immunoglobulines et d'autres séquences activatrices. L'expression du gène E2A semble ubiquitaire. La structure protéique de E12/E47 les apparente à une famille de protéines se liant sous forme de dimères à l'ADN grâce à des motifs "hélice-bouclehélice ". Dans ces molécules, l'hypothèse est que la dimérisation est assurée par deux hélices $\alpha$ séparées par une boucle peptidique alors que la région interagissant avec l'ADN correspond à une séquence riche en acides animés basiques, adjacente au motif de dimérisation [4]. Outre E12/E47, les produits des oncogènes myc et les facteurs " différenciateurs" myogéniques que sont MyoD1, Myf5, myogénine et, plus récemment caractérisé [5, 6], Myf 6 (aussi appelé MRF4 et herculine) appartiennent à cette famille et sont capables de former des hétéro- dimères entre eux et avec les produits du gène $E 2 A$ [7]. Le point de cassure dans le gène $E 2 A$ passe, chez les malades atteints de leucémies avec translocation $\mathrm{t}(1 ; 19)$, en amont de la région codant pour le domaine " hélice-boucle-hélice ", si bien que la région responsable de la dimérisation et de la liaison à l'ADN n'est pas retenue sur le chromosome 19. En revanche, le fragment d'ADN en provenance du chromosome 1 , fusionné à la partie 5' du gène $E 2 A$, code pour une protéine possédant une boîte homéo. Ces motifs peptidiques sont du type "hélice-tourhélice " bien caractérisé au niveau de facteurs de transcription procaryotiques dont l'archétype est le répresseur du phage $\lambda\left(\mathrm{m} / \mathrm{s}\right.$, suppl. au $n^{\circ} 1$, vol. 5, p. 33). Ils sont présents au niveau des produits de gènes intervenant dans le développement de la drosophile (gènes homéotiques) et des mammifères (homéogènes), ainsi que de facteurs de transcription caractérisés chez l'animal: facteurs hypophysaire Pit 1 , hépatique HNF1, protéines oct1 et oct2 se fixant sur les motifs octamères de nombreux promoteurs et enhancers $\left(\mathrm{m} / \mathrm{s} n^{\circ} 3\right.$, vol. 5, p. 172).

La translocation $\mathrm{t}(1 ; 19)$ a donc amené à la création d'un gène hybride dont environ la moitié 5' est constituée du gène $E 2 A$ et la moitié 3' d'un gène normalement localisé sur le chromosome 1 et codant pour un probable facteur de transcription; ce gène a été appelé $\mathrm{Prl}$ (pre-B leukemia). La séquence de fixation à l'ADN de la protéine hybride est celle de $P r l$ et on peut supposer que $E 2 A$ contribue à son activité par son domaine d'activation transcriptionnelle. Le gène $\mathrm{Prl}$ normal ne semble pas exprimé dans les cellules pré-B, alors que l'expression du gène hybride $E 2 A-P r l$ est forte dans les cellules

leucémiques 
ayant la translocation $(1 ; 19)$. Le mécanisme de la transformation cellulaire pourrait donc n'être que le reflet de cette expression forte et anormale d'une protéine E2A-Prl sinon de même effet et de même spécificité que la protéine Prl normale. L'existence d'un domaine activateur E2A pourrait aussi changer la spécificité de $\mathrm{Prl}$, soit en augmentant son efficacité, soit même en transformant un inhibiteur en stimulateur transcriptionnel. Dans tous les cas, on peut ainsi faire l'hypothèse que la croissance cellulaire excessive des cellules leucémiques pré-B est liée à la stimulation anormale des gènes normalement contrôlés par le produit de Prl. Il est possible que le clonage moléculaire de sites de réarrangements chromosomiques fréquents dans d'autres types de proliférations malignes démontre que, en fait, la création de telles molécules chimères oncogéniques est un phénomène non exceptionnel dans les cancers humains. Plusieurs exemples en sont connus dans des tumeurs solides, un nouveau venant d'être rapporté dans des cancers papillaires de la thyroïde par des équipes italiennes [8]. Il s'agit de la recombinaison du produit d'un proto-oncogène codant pour une tyrosine kinase (gène ret) avec la région 5' d'une autre protéine. Ce qui pourrait être rare, en fait, c'est l'activation d'un oncogène sans modifications qualitatives associées de son produit, qu'il s'agisse d'une délétion partielle, d'une mutation ponctuelle ou de la constitution de molécules chimères. Un des rares exemples connus où seule l'anomalie de régulation de l'expression du gène est en cause est, à ce jour, celui des oncogènes myc.

A.K.

1. Kaplan JC, Szajnert MF. Chromosomes et cancer. médecine/sciences $1985 ; 1: 17-23$.

2. Nourse J, Mellentin JD, Galli N, et al. Chromosomal translocation $\mathrm{t}(1 ; 19)$ results in synthesis of a homeobox fusion mRNA that codes for a potential chimeric transcription factor. Cell 1990 ; 60 : 535-45.

3. Kamps MP, Murre C, Xiao-Hong S, Baltimore D. A new homeobox gene contributes the DNA binding domain of the $\mathrm{t}(1 ; 19)$ translocation protein in pre-B ALL. Cell $1990 ; 60$ : 547-55.
4. Murre C, McCaw PS, Baltimore D. A new DNA binding and dimerization motif in immunoglobulin enhancer binding, daughterless, myoD and myc proteins. Cell 1989 ; $56: 777-83$.

5. Rhodes SJ, Konieczny SF. Identification of MRF4 : a new member of the muscle regulatory factor gene family. Genes Dev $1989 ; 3$ : 2050-61.

6. Miner JH, Wold B. Herculin, a fourth member of the MyoD family of myogenic regulatory genes. Proc Natl Acad Sci USA $1990 ; 87: 1089-93$

7. Murre C, McCaw PS, Vaessin H, et al. Interaction between heterologous helix-loophelix proteins generate complexes that bind specifically to a common DNA sequence. Cell 1989 ; 58 : 537-44

8. Grieco M, Santoro M, Berlingieri MT, et al. PTC is a novel rearranged form of the ret protooncogene and is frequently detected in vivo in human thyroid papillary carcinomas. Cell $1990 ; 60$ : 557-63.

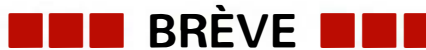

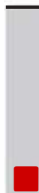

\section{possibilite deur sur mesure. La} spécifiques pour une molécule donnée pcut avoir des applications industriclles importantes. En attendant les retombées des efforts actuels dans le domaine de la modélisation moléculaire, d'autres approches plus empiriques sont tentées. Un article récent [1] décrit des conditions expérimentales permettant de transformer l'albumine en un récepteur relativement spécifique de moléculcs organiques telles que l'acide parahydroxybenzoïque. Ces conditions n'ont rien de biologique puisque de fortes concentrations de protéine et du composé organique sont mélangécs, permettant à ce dernier de laisser une " empreinte " sur la protéine. Tant que cette protéine est maintenuc dans un milieu organique, elle garde la mémoire du composé et en devient un récepteur spécifiquc. L'activité réceptrice disparaît complètement dans un milicu aqueux en raison de la perte de la rigidité de la protéine. Cette approche pourrait trouver des applications industrielles pour la purification de composés biologiques et dans le domaine de la biocatalysc. [1. Braco L, et al. Proc Natl Acad Sci USA 1990 ; 87 : 274-7.]
BRÈVE

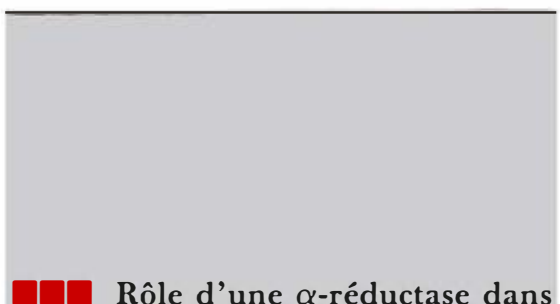

la polykystose ovarienne? Lc syndrome des ovaires polykystiques, caractérisé par des règles irrégulières ct un hirsutisme, est unc causc commune de non-fertilité anovulatoire. Les principaux signes biochimiques en sont une élévation de l'hormone lutéinisante et des androgènes. Chez 11 malades atteintes de ce syndrome, dont les activités de la 21-hydroxylase et de la $3 \beta$-hydroxystéroïdc déshydrogénasc étaicnt normales, unc équipc écossaisc [1] a trouvé un rapport augmenté des dérivés $5 \alpha$ aux dépens des $5 \beta$ qui fìt penser à un excès d'activité de la $5 \alpha$-réductase. Cette enzyme transforme le cortisol en $5 \alpha$ dihydrocortisol, mais aussi la testostérone en dihydrotestostérone, beaucoup plus active en tant qu'hormone mâle que la testostérone. Une revue sur l' $\alpha$-réductase, par Mauvais-Jarvis et al., a paru dans $\mathrm{m} / \mathrm{s}$ en $1986\left(n^{\circ} 5\right.$, vol. 2, p. 267). Elle montrait l'origine privilégiée de cette enzyme dans le foie et la pcau. On savait déjà que son déficit est cause d'une forme de pscudo-hermaphrodisme, et son excìs d'un hirsutisme, mais la notion de son rôle dans les ovaires polykystiques paraît entièrement nouvelle. Ces travaux conduisent à insister sur deux points importants: dans le domaine des polykystoses ovariennes, l'attention s'était jusqu'à maintenant portéc sur les ovaires cux-mêmes, la surrénale, source d'androgènes, et le couple hypophyse-hypothalamus; si l' $\alpha$-réductase joue un rôlc majeur, ce pourrait être en réalité une maladie du foic et de la peau, sources principales de l'enzyme. Sur un plan plus général, alors que les recherches modernes ont tendance à approfondir le rôle des hormones et des réceptcurs, il ne faut pas oublier celui des enzymes, dont le fonctionnement insulfisant ou excessif peut perturber l'ensemble des régulations.

[1. Stewart PM, et al. Lancel 1990 i : 431-3.] 
Des chélateurs oraux du fer dans la surcharge ferrique. Se débarrasser du métal en excès est un impératif dans les affections qui comportent une surcharge en fer. La saignéc, efficace dans les hémochromatoses idiopathiques, est impossible dans les maladies anémiantes, comme les thalassémies et les anémies aplastiques, qui réclament des transfusions. On dispose depuis près de trente ans d'un médicament efficace, la desferrioxamine; celle-ci toutefois doit être administrée en injections répétécs pendant des années et est très coûtcuse. On a donc cherché des agents actifs par voie orale qui soient dépourvus de toxicité. Un composé de la famille des hydroxypyridones, le 1,2-diméthyle-3-hydroxypyridine4-one (LI) a été étudié en détail par une équipe anglaise [1]. L'efficacité du produit, testé jusqu'à présent sur environ 150 patients, paraît certaine. Cependant des études sur le rat et la souris ont montré une certaine toxicité (baisse du taux des globules rouges et blancs) et, surtout, on a signalé un cas d'agranulocytose qui a failli être mortel [2]. Aussi un éditorial de Lancet [3] suggérait-il d'abandonner le LI. Un autre son de cloche est en revanche fourni par des auteurs de Bombay [4] : ils font valoir que dans leur pays la desferrioxamine n'est accessible qu'à $5 \%$ des malades qui en auraient besoin. Ils dressent, en face de l'éventualité rare d'un accident hématologiquc, la possibilité salvatrice du LI en faveur de nombreux malades. Ils plaident donc pour la poursuitc du traitement, au moins dans les pays où il est le seul possible, en attendant que l'on ait trouvé des dérivés oraux moins toxiques, ce que l'on peut espérer pour un avenir proche, tant dans la série des pyridinones que dans celle dont fait partie la desferrioxamine [5].

[1. Kontoghioghes GJ, et al. Lancel 1987 ; i : 1294-5.]

[2. Hoffbrand AV, et al. Lancet 1988 ii : 457.]

[3. Éditorial. Lancel 1989 ; ii : 1016-7.]

$\mathrm{m} / \mathrm{s} n^{\circ} 5$ vol. 6 , mai 90
[4. Agarwal MB. Lancet 1990 ; i : 601.

[5. Porter JB. Eur J Hematol 1989 ; $43:$ 271-85.

$\square$ Des astrocytes føtaux redonnent sa jeunesse à un cerveau adulte. Pendant une période du développement post-natal, les circuits nerveux du cortex cérébral responsables de l'intégration des influx venus de la rétine sont susceptibles de sc modifier en fonction de l'expérience (voir lexique $n^{\circ} 4$, vol. 6, p. 388 , " modifications structurales liées à l'activité "). Si un des deux yeux est maintenu clos quelques mois après la naissance avant d'être ouvert (chez le chaton), les neurones corticaux perdent la possibilité d'être activés à partir des deux yeux. Durant trois mois, cette perte est réversible si l'œil est réouvert, grâce à la plasticité des systèmes de connexions nouvellement établis ; en revanche, dépassée cette période critique, la perte de la binocularité cellulaire devient irréversible. Ou du moins, c'était ce que l'on pensait jusqu'à ce que Müller et Best [1] démontrent que l'on pouvait induire une nouvelle phase de plasticité des circuits nerveux dans le cortex cérébral du chat adulte. Pour ce fairc, ils ont implanté dans la région occipitale du cortex - qui contient les centres visuels - des astrocytes immatures issus du cortex de chatons nouveau-nés. Cette transplantation (au contraire de transplantations contrôle d'autres types de tissus) a donné une nouvelle chance de récupération de la binocularité cellulaire à des chats qui en avaient été privés par occlusion d'un œil au-delà de la période critique. Le système de connexions nerveuses adulte a en effet repris une manifeste souplesse et la binocularité cellulaire s'est établie. $\mathrm{La}$ plasticité du système nerveux central de l'adulte avait surtout été démontrée, jusqu'à présent, dans les réorganisations consécutives à des lésions de certains de scs éléments propres après atteinte neurodégénérative ou section de fibres. La démonstration faite ici élargit considérablement ce champ puisque, d'une part, il s'agit d'une réponse induite sans lésion propre et que, d'autre part, la plasticité retrouvée est le moyen d'unc véritable thérapeutique aux conséquences fonctionnelles vérifiables. [1. Müller CM, Best J. Nature 1989 ; 342 : 427-30.]

L'acatalasémie. Le déficit en catalase ou acatalasémie est une affection réccssive autosomique, souvent asymptomatique, mais qui parfois provoque une nécrose buccale. On en connaît deux types principaux, japonais et suisse. Dans le type suisse, le déficit cst limité aux globules rouges, l'instabilité de la molécule étant compensée par la synthèse protéique dans les tissus actifs. La modification de la migration électrophorétique de l'enzyme résiduelle fait penser qu'il s'agit d'une mutation ponctuelle, dont la nature n'a pas été encore précisée à notre connaissance. Au contraire le type japonais n'a qu'une activité résiduelle de $1 \%$, la même dans les érythrocytes et les tissus, et qui semble posséder des propriétés normales. Le gène de la catalase a été cloné en 1986 [1]. Il porte 13 exons et code pour une protéine de 526 acides aminés. Wen et al. (Nagano et Okayama, Japon) ont cloné le gène d'un sujet déficient [2] ; ils ont trouvé sept différences avec la séquence de référence, dont la plupart sont dues à des polymorphismes. La mutation significative est le remplacement d'un $G$ par un A en position 5 de l'intron 4 , ce qui supprime le site d'épissage en $5^{\prime}$ de cet intron. Ce défaut d'épissage a été démontré en utilisant une construction de gènes chimériques contenant la portion en cause du gène de la catalase, liée à l'exon 3' de la globine, exprimés dans des cellules COS-7. Il est donc probable que cette mutation de l'intron 4, retrouvée chez un autre malade japonais non apparenté, cst bien à l'origine de l'acatalasémie japonaise.

[1. Quan F, et al. Nucleic Acids Res 1986 ; 14 : 5321-35.]

[2. Wen JK et al. J Mol Biol 1990 ; 211: 383-93.]
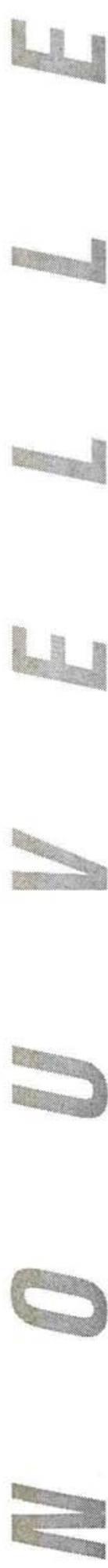
Récepteurs pour les complexes anticorps/HIV et synergies virales. Le réccpteur le micux connu du virus du SIDA (HIV) à la surface cellulaire est la protéine CD4. Les complexes antigène/anticorps, et donc les particules virales liécs à des anticorps spécifiques, peuvent se fixer à d'autres molécules membranaires, principalement les réccptcurs pour les fragments $F c$ des immunoglobulines (R-Fc) et les réccptcurs du complément. Les molécules R-Fc sont induites sur les monocytes ct d'autres ccllulcs par des cytokines et en réponse à des infections virales par des agents de la famillc herpès, notamment le cytomégalovirus (CMV). De fait, in vitro, des fibroblastes préalablement infectés par le CMV peuvent être surinfectés par HIV complexé à des anticorps, même en présence d'anticorps anti-CD4. En revanche, le prétraitement des cellules par des immunoglobulines, saturant les R-Fc, prévient l'infection ultérieure par HIV. Des fibroblastes non infectés par le CMV n'expriment pas le R-FC ct sont résistants à l'infection par HIV. On pcut supposer que, après pénétration dans la cellule, HIV verra aussi sa pathogénicité exaccrbée par la co-infection avec un virus herpès du fait de la transactivation des séquences régulatrices du LTR par des facteurs transcriptionnels du type $N F-\kappa B$ qui sont eux-mêmes activés lors de l'infection par le virus herpès. Nul ne sait au juste si de tels phénomènes d'infection de diverses cellulcs via les réceptcurs $\mathrm{Fc}$ survicnnent in vivo. Cette éventualité, au moins théorique, renforce cependant encore la prudence avec laquelle devront être entrepriscs les tentatives de vaccination puisque, comme nous l'avons déjà signalé, les anticorps engendrés peuvent parfaitement être facilitants et non neutralisants, permettant une infection de cellules $\mathrm{CD}^{-}{ }^{-}$. De plus, les résultats du laboratoire de R. Weiss (Londres, GB) donnent une nouvelle explication virale déjà bien documentés chez les malades atteints du SIDA.

[1. McKeating JA, et al. Nature $1990 ; 343:$ 659-61.]

Des anticorps peuvent se développer contre du GM-CSF administré en thérapeutique. Lc gène du facteur stimulant les colonies de granulocytes et macrophages (GMCSF) a été cloné. On en a tiré des recombinants, et lcur utilisation fonctionnelle a été exploréc avec des résultats préliminaires encourageants depuis deux ans. Dans le colibacille, la production de GM-CSF fournit unc protéine non glycosylée; dans la levure, elle est $\mathrm{N}$-glycosylée, et dans des cellules de mammifères (cellules $\mathrm{CHO}$, dérivées d'ovaires de hamster chinois), à la fois les sites $\mathrm{N}$ - et $\mathrm{O}$ sont glycosylés, comme dans la protéine native. L'efficacité des trois formes s'est montréc la même dans des essais cliniques en phases I et II. Une équipe anglaise [1] vient de montrer que des anticorps pouvaient apparaître au cours du traitement. Dans leur essai clinique, ils ont utilisé la forme synthétisée dans la levure; les malades étaient des porteurs de tumeurs solides ou des maladies de Hodgkin réfractaires au traitement. Sur 16 sujets ayant reçu le traitement, quatre virent apparaître des anticorps en une semainc. Leur sérum réagissait contre le GM-CSF " de levure ", mais aussi contre celui de "Coli" ; en revanche, il n'avait pas d'action contre celui qui était dérivé d'une culture de mammifere. Des expériences de déglycosylation élective du GM-CSF natif ont confirmé que les anticorps étaient dirigés contre une partie de la molécule normalement protégéc par des liaisons O-glycosyles. Ces anticorps peuvent-ils être nocifs? en culture de moelle on n'a pas pu mettre en évidence d'action neutralisante des anti- corps. In vivo, il semble que la préscnce d'anticorps chez des malades ait accéléré la clairance du GM-CSF. Il est donc important de préciscr la fréquence du phénomène, de survciller l'apparition d'anticorps chez certains malades malgré le traitement immunosupresscur, et finalement, sans doutc, d'avoir recours si possible au produit dérivé de cellules de mammifères.

[1. Gribben JG, et al. Lancel 1990 ; i : 434-7.]

Une cytokine inhibitrice de la prolifération des cellules souches hématopoietiques. Unc cytokine, antéricurement dénommée MIP $1 \alpha$ (macrophage inflammatory protein $1 \alpha$ ) vient d'être re-découverte sous le nom de SCI (Stem cell inhibitor) par des chercheurs britanniques (Glasgow, Oxon) et américains (Cambridge, MA, USA) étudiant un inhibitcur de la prolifération des cellules souches de la moelle osseuse [1]. Cette protéinc de 8000 daltons, formant probablement des agrégats in vivo, est active à très basse concentration; clle bloque l'entrée dans le cycle cellulaire des CFU-S (colony forming units-stem cells). D'autres inhibiteurs de croissance des cellules précurscurs des lignées hématopoïétiques ont été décrits, notamment un tétrapeptide découvert et analysé par E. Frindel [2] ; ils sont nettement différents de la cytokine SCI/MIP $1 \alpha$. Ces molécules renforcent la notion qu'aux facteurs de croissance doivent correspondre, peut-être en nombre équivalent, des inhibiteurs de croissance dont l'intérêt thérapeutique pourrait être grand, qu'il s'agisse de ralentir la prolifération d'un tissu cible tumoral, ou de mettre au repos les cellules souches afin de diminuer la toxicité d'une chimiothérapic anticancéreusc et ainsi les risques d'aplasic médullairc thérapeutique sévèrc. [1. Graham GJ, et al. Nature 1990 ; 344: 442-4.]

[2. Frindel E, Guingon M. Expl Hematol 1977 ; 5 : 74-6.] 